\title{
Should Dental Schools Train Dentists to Routinely Provide Limited Preventive Primary Medical Care? Two Viewpoints
}

\author{
Viewpoint 1: Dentists Should Be Trained to Routinely Provide \\ Limited Preventive Primary Care
}

Donald B. Giddon, DMD, PhD; R. Bruce Donoff, DMD, MD

\section{Viewpoint 2: Dentists Should Be Trained in Primary Care Medicine to Enable Comprehensive Patient Management Within Their Scope of Practice}

\begin{abstract}
Paul C. Edwards, MSc, DDS; Lawrence I. Goldblatt, DDS, MSD
Abstract: This Point/Counterpoint acknowledges the transformation of dental practice from a predominantly technically based profession with primary emphasis on restoration of the tooth and its supporting structures to that of a more medically based specialty focusing on the oral and maxillofacial complex. While both viewpoints accept the importance of this transformation, they differ on the ultimate desired outcome and how changes should be implemented during training of dentists as oral health professionals. Viewpoint 1 argues that, in response to a shortage of both primary care providers and access to affordable oral health care, dentists need to be able and willing to provide limited preventive primary care (LPPC), and dental educators should develop and implement training models to prepare them. Among changes proposed are consideration of three types of practitioners: oral physicians with sufficient training to provide LPPC; dentists with excellent technical proficiency but minimal medical and surgical training; and mid-level providers to provide simple restorative and uncomplicated surgical care. Viewpoint 2 argues that the objective of dentists' education in primary care medicine is to help them safely and effectively provide all aspects of oral health care, including appropriate preventive medical care, that already fall within their scope of knowledge and practice. Dental educators should encourage students to use this knowledge to take full ownership of non-tooth-related pathologic conditions of the oral and maxillofacial complex not currently managed in the dental setting, but encouraging graduates to expand into nondental LPPC outside the recognized scope of practice will only further exacerbate fragmentation of care.
\end{abstract}

Dr. Giddon is Professor of Developmental Biology Emeritus, Harvard School of Dental Medicine; Dr. Donoff is Dean, Harvard School of Dental Medicine; Dr. Edwards is Professor, Department of Oral Pathology, Medicine, and Radiology, Indiana University School of Dentistry; and Dr. Goldblatt is Dean Emeritus and Professor Emeritus, Department of Oral Pathology, Medicine, and Radiology, Indiana University School of Dentistry. Direct correspondence to Dr. Donald B. Giddon, Harvard School of Dental Medicine, 190 Longwood Ave., Boston, MA 02115; donald_giddon@hms.harvard.edu.

Keywords: dental education, dentistry, access to health care, curriculum, health care systems, scope of practice, interprofessional relations, interprofessional education, primary care, preventive primary care

Submitted for publication 11/4/16; accepted 12/2/16

doi: $10.21815 / J D E .016 .023$

$\mathrm{T}$ This Point/Counterpoint acknowledges the transformation of dental practice from a predominantly technically based health profession with a primary emphasis on restoration of the tooth and its supporting structures to that of a more medically based specialty focusing on the oral and maxillofacial complex. While both viewpoints accept the importance and significance of this transformation, they differ on the ultimate desired outcome and how those necessary changes should be implemented during training of dentists as oral health professionals, specifically regarding whether dental students should be trained to routinely provide limited preventive primary care. 


\section{Viewpoint 1: Dentists}

Should Be Trained to

Routinely Provide Limited

Preventive Primary Care

This viewpoint is based on the argument that dentists should routinely provide limited preventive primary care (LPPC) (including chairside screening for chronic disease and monitoring compliance with prescribed medications) for the following reasons. The training of oral health care professionals must adjust to the changing health care needs of an aging population of patients with more complex medical diseases. ${ }^{1}$ Also, dentists should be better prepared to provide comprehensive care for patients because dentistry cannot be separated from medicine. ${ }^{2,3}$ Historically, Dean Alfred Owre of Columbia and others defined it as a specialty of medicine, in contrast to Dr. William J. Gies who strongly supported dentistry "as a separately organized profession [but one that] should be made the service equivalent of an oral specialty of the practice of medicine." ${ }^{\prime 4}$ Furthermore, identifying previously undiagnosed chronic diseases in the dental office (such as diabetes, ${ }^{5}$ hypertension, ${ }^{6}$ hypercholesterolemia, ${ }^{6}$ and cardiovascular disease ${ }^{7}$ ) can reduce morbidity and health care costs. ${ }^{6-8}$ Similarly, orthodontists, who see patients once a month for two years during their most formative period, can reduce health care costs by recognizing and referring patients with developmental, eating, and behavioral disorders. $^{9}$

Another reason underlying our argument is the maldistribution of health care resources in the U.S. with an estimated shortage of primary care physicians of 40,000 by $2020,{ }^{10}$ as well as significant deficiency of access to affordable dental care, due in part to the financial lure of specialization to both dentists and physicians. ${ }^{11}$ An opportunity-almost an imperative - now exists for dentists to help fill these gaps by participating in greater integration and collaboration with all health professions. A final reason is that patients have been found to support involving dentists in their overall health, taking advantage of visiting their dentist more often than their physician. ${ }^{12}$ Greenberg et al., for example, found that patients were favorably inclined toward dentists' doing chairside screening for chronic diseases. ${ }^{13}$

\section{What Changes Are Needed to Train Dentists to Provide LPPC?}

In the history of dentistry, its relationship to the medical profession has varied considerably, from dentists' being called barber surgeons, tooth doctors, or physicians of the mouth ${ }^{14}$ to the current mission of Harvard School of Dental Medicine "to transform dentistry by removing the distinction between oral and systemic health." To train dentists to provide LPCC, U.S. dental schools will have to determine what should be taught, when, and by whom to provide these expanded services and what should be the criteria for recruiting and training the teachers. Unfortunately, our perception is that most current predoctoral dental curricula focus medicine and surgery education in the first two years with little reinforcement or opportunity for integration with clinical training in the final two years, while other schools provide only enough medical and surgical training in the first year-plus to satisfy Commission on Dental Accreditation (CODA) requirements. Examples of greater integration throughout the four years were recently presented by Harvard and the University of Washington; in those dental schools, dental faculty work with medical school faculty throughout the curriculum to prepare graduates to provide LPPC. ${ }^{15}$ Whatever changes are made, the new educational paradigm must include re-emphasizing that dentistry cannot be separated from medicine, at the same time reminding ourselves that the mouth and contiguous area are the most important parts of the body, with their role in survival through intake of food, water, and air; socialization, including communication of content by the teeth and tongue; and affect with the muscles of facial expression. ${ }^{16}$

Given the variations in abilities and interests among oral health providers to acquire the appropriate knowledge and skills to provide expanded healthrelated services, we believe there is a need to consider the training of three types of oral health providers. ${ }^{15-17}$ These three are oral physician, ${ }^{18,19}$ dentist, and midlevel provider (MLP) - an expansion upon the two tiers recently described by Guay. ${ }^{20}$ Oral physicians would become qualified as dentists with sufficient training in oral medicine and surgery to provide dental care and LPPC by screening for chronic diseases and monitoring medications, while overseeing all oral health services whether provided by dentists or nondentists, including MLPs. Degree options for oral physicians could include DMD/DDS+general 
practice residency, DMD/DDS+MD/DO, MD/ $\mathrm{DO}+\mathrm{DMD} / \mathrm{DDS}+$ residency, and MD+postdoc. Dentists in this three-tier model would offer excellent technical proficiency but with minimal medical and surgical training, and MLPs would deliver simple restorative and uncomplicated surgical care.

Under this model, predoctoral students who intend to become oral physicians would be considered oral medicine specialists in training and, as such, would have didactic and clinical experiences in medicine and surgery reinforced throughout their four years and integrated with preparation for dental practice. Also facilitated would be opportunities for cross-training with dental and medical student rotations and clerkships or internships/general practice dental residencies; examples might include case presentations shared by orthodontics, psychiatry, pediatric dentistry, and obstetrics/gynecology.

\section{Overcoming Barriers to Train Dentists to Provide LPPC}

Dental profession. Perhaps the greatest barrier to implementing this plan would be the dentists themselves who are reluctant to forgo their very successful business plan, with little accountability for quality or appropriateness of their services. ${ }^{21}$ To accept increased health care responsibilities, oral physicians would have to incur additional insurance and training costs without commensurate reimbursement. Such concerns may be age-related, with older dentists more willing to undertake additional training than younger dentists who are still concerned about debt reduction..$^{22}$

Mid-level providers. The role of MLPs in training dentists to be able to provide LPPC would differ somewhat from their use in medical training and practice. In contrast to dentistry, in which the use of MLPs is essentially controlled by organized dentistry, except for some recent activity by both dental hygienists and dental therapists to practice autonomously, ${ }^{23}$ medical MLPs (physician assistants, nurse practitioners) have been aggressively seeking to add to their required education, degrees, and certification to enhance their professional and financial status. ${ }^{24}$ For the most part, these developments are responding to market forces, such as the lower costs of MLPs without sacrificing safety, ${ }^{25}$ with an additional financial benefit to educational institutions for providing the required additional training.

The influence of market forces supporting the use of medical MLPs to decrease the cost of health care should not go unnoticed by the dental profession. For example, with the increasing public perception that dental care is more costly than it would be if MLPs performed routine procedures, ${ }^{26}$ it may not be too long before the public does not distinguish between dentists and dental therapists, who like medical MLPs may soon increase their status and employment opportunities at higher salaries in corporate settings than they would receive in private dental practice. ${ }^{27}$ This possibility provides yet another reason for dentists to add the professional role of oral physician, which better connotes what they can and should do at the top of the hierarchy of oral health providers involved in the integrated health care of their patients.

Economic factors. There are several economic considerations associated with implementing these proposed changes in training dentists to provide LPPC while ensuring access to affordable dental care. These include costs of developing new curriculum models; compensation for medical screening in a dental setting; costs associated with ensuring compatibility and accessibility of shared medical and dental records; debt relief for those willing to provide LPPC; and cost benefits of reallocation of the health care workforce involving the greater use of MLPs such as dental therapists to significantly decrease costs of dental care and free dentists to function as oral physicians able to provide LPPC while being available for what only he or she can do.

Whether it is more cost-effective to train dentists to provide LPPC or increase the number of primary care physicians has yet to be determined. Alternative, less costly solutions for dentists to become involved in LPPC may be to increase their training in how to utilize medical MLPs such as nurse practitioners or physician assistants in the dental practice. Those medical MLPs may be able to be compensated directly or indirectly through a doctor's office, if and when dentists can receive compensation for medical procedures performed in a dental office, given that technically dentists are already categorized as physicians under Medicare. ${ }^{28}$

Cultural differences. Despite universities' encouragement of development and evaluation of new educational and training models including basic medical and behavioral sciences, dental education has continued to focus on technology. In dental education, Rosebury noted, "Experimental science [now probably evidence-based research] . . . is taught better than it used to be, . . but with some noteworthy exceptions, its spirit has not yet caught 
on in dental students, in dental clinical teachers, or in dental practitioners, as it has in their counterparts in medicine. It is necessary to learn something about science to be a dentist, but it is still possible to forget nearly all of it and yet be a successful practitioner or, even in some areas, a successful dental teacher." ${ }^{29}$ In essence, dental graduates of today are the products of their mentors, who may not agree that dentistry cannot be separated from medicine.

\section{Preparing the "Compleat" Health Professional}

We are again at a crossroads as a profession in the relationship between dental and medical education, leaving hope for change to the only reliable engine for innovation: the universities, of which all U.S. dental schools are now a part. Beginning in 1867 , the university's role in training dentists at Harvard was a major factor in helping students develop the enlightened perspective essential for becoming the "compleat" health professional. ${ }^{2}$ In contrast to the U.S., dental and medical training in Europe and elsewhere is much more vertical and focused on professional development immediately after graduation from high school or the "gymnasium" with little opportunity for broader education and experience available in the U.S. educational system. Unfortunately, despite our efforts to take advantage of a diverse educational experience together with involving dentists in primary care, there is a competing trend focused on greater technical specialization of dental graduates with minimal medical competence.

Consequently, the call for reform may not become a clarion call until it emanates from the university pulpit. Where else but in university-based programs would we have the freedom to experiment with new health care systems, as at the University of Minnesota, ${ }^{26,30}$ where the efficacy of MLPs is being developed and evaluated scientifically rather than being assessed politically by organized dentistry? ${ }^{31}$ Similarly, in 2015 the Virginia Commonwealth University School of Dentistry, partnering with government agencies for funding innovative training models, "received a \$2.4 million federal grant [to] help initiate a curriculum that integrates interprofessional education and collaborative care into the pediatric dentistry residency training program. ${ }^{\prime 32}$ Whatever the resulting changes are for training dentists to be able to provide both oral and primary care services will depend on interprofessional collaboration among and between these members of the health care workforce, reallocated by function rather than constrained by traditional professional barriers between them.

This viewpoint provides evidence supporting the argument that dentists should be trained to provide patient-centered LPPC, beginning with their predoctoral education, while their training in oral medicine prepares them to recognize the oral manifestations of systemic disease, as well as perform chairside screening for chronic disease. To be consistent with a university-based professional education, dental curricula need to be modified to reinforce and integrate didactic and clinical training in medicine and surgery, including physical, oral, and mental health essential for obtaining and maintaining patients' physical, oral, and mental health. This model includes the possible need for a three-tiered level of training: oral physician, dentist, and midlevel provider, distinguished in part by the extent of medicine and surgery in the respective curricula or the emphasis on biotechnology. Regardless of what curricular changes do occur, dentistry cannot be separated from medicine if we are to be able to better serve our patients by being able to provide LPPC as well as accessible, affordable oral health care.

\section{Viewpoint 2: Dentists Should Be Trained in Primary Care Medicine to Enable Comprehensive Patient Management Within Their Scope of Practice}

This viewpoint agrees that we should be training dentists to provide limited preventive primary medical care, but argues that should be only in the context of management of each individual patient and within the recognized scope of dental practice. The goal must be to graduate dentists who have both a solid theoretical and practical knowledge of primary care medicine, such that they are able to safely and effectively provide all aspects of treatment, including preventive medical care that is within the scope of their training. However, we see no logical rationale for suggesting that this knowledge base should translate to dentists' routinely providing primary preventive medical care outside our scope of 
practice. Instead, we believe that there already are a large number of conditions of the head and neck structures clearly within the scope of our education that primary dental providers are best suited to manage but that are nevertheless being referred out to non-dental providers. Before expanding outside our scope of practice, we must first empower our dental graduates to take full ownership of those neglected areas that are already within our expertise. ${ }^{33}$

The authors of Viewpoint 1 argue that we should expand the scope of dental practice beyond the traditional confines of the oral and maxillofacial structures, while others have proposed that we transition from using the designation "dentist" to variations on "oral physician." "1,8,15,18 Although we can argue the benefits of using titles such as "dentist," "dental surgeon," or "oral physician" or the rationale for offering a DMD versus DDS degree, in the end the title is of little consequence (provided, of course, that such titles do not inadvertently mislead the public). Ultimately, what is germane is validating our competence, as a profession, within our scope of practice.

\section{Preventive Care Within Current Scope of Practice}

As it now stands, dental care providers already routinely provide three broad categories of preventive care. Primary preventive care (risk reduction), comprising measures used to prevent new disease cases by controlling modifiable risk factors, commonly focuses on patient education. Examples include counseling on smoking cessation to reduce the risk of developing periodontal disease and head and neck cancer, dietary counseling on preventing dental caries, advocating for use of mouth guards in contact sports, promoting community water fluoridation, and counseling on safe sex practices and advocating for (but not administering) immunizations to reduce the risk of developing high risk human papilloma virus (HPV)-associated cervical and oropharyngeal cancer. Secondary prevention, representing early detection of unrecognized disease through screening, is often, but not exclusively, targeted at defined higher risk populations. Examples include early detection of head and neck cancer by means of a thorough examination. Finally, tertiary prevention focuses on reducing the morbidity of existing disease in order to diminish the likelihood of complications. This includes followup computed tomography scans in patients treated for oral cancer to identify recurrences at an early stage, and intensive blood glucose monitoring in the diabetic patient to reduce diabetes-related complications, such as periodontal disease and delayed wound healing. Although arguments can be made as to the risk-benefit ratios and cost-effectiveness of secondary and tertiary screening, ${ }^{34,35}$ there is no doubt that primary disease prevention must remain a principal focus of the dental profession.

\section{Challenges to Expanding Dental Scope of Practice}

Some of our well-meaning colleagues have argued anecdotally that dentists should be ordering routine blood work and diagnostic tests (e.g., CBC and comprehensive metabolic panels) as part of an overall comprehensive health assessment, even in the absence of any signs or symptoms to suggest a possible concern affecting the dental management of our patients. Arguments in support of an expanded role for dentists in the health care system focus on the obvious fact that we are first and foremost health care professionals and therefore should not focus exclusively on the oral cavity. ${ }^{36}$ Moreover, we are all cognizant of the reality that oral diseases can have a significant impact on our patients' overall health and well-being. The opposite is also true: patients' overall health status can have a direct bearing on their oral health status, response to dental therapy, and ability to undergo needed treatment. As a result, we are all in agreement that the graduating dentist must be fully versed at recognizing and managing the complete spectrum of health concerns, ordering the most appropriate diagnostic tests, and assessing all information required to properly diagnose and safely treat all conditions of the oral and maxillofacial region that are within our scope of expertise and training. But ordering routine blood work and other diagnostic tests for patients, absent any signs or symptoms suggestive of an underlying condition that could directly impact our management of conditions of the oral and maxillofacial complex, is an obvious non sequitur.

Moreover, unless the dentist is prepared to coordinate the long-term definitive management of any non-oral and maxillofacial medical conditions discovered as a result of this testing, such as hyperlipidemia or hypertension, the dentist is mandated to refer the patient back to his or her primary care physician for appropriate follow-up. Is the dentist in this situation prepared to accept any potential liability resulting from ordering inappropriate or unnecessary diagnostic tests? What about the other scenario, in 
which the dentist, having reviewed a panel of routine test results that appear to be within the normal range, fails to recommend follow-up with the patient's primary medical provider for comprehensive medically appropriate assessments? When a dental provider orders selective screening tests, whether appropriate or not for the patient's risk status and medical history, that patient cannot help but conclude that he or she no longer needs to see a primary care physician. If this patient subsequently develops a condition such as breast or prostate carcinoma, which consequently is not diagnosed at an earlier, more treatable stage, would this not legitimately represent a failure to diagnose?

One argument we have heard proffered by advocates who believe that dental providers should be providing primary preventive medical care is that dentists already assess their patients' systemic health, such as measuring blood pressure. While true, this argument overlooks the fact that the rationale for assessing vital signs such as blood pressure relates directly to our scope of practice: evaluating whether our patient is capable of safely tolerating the proposed treatment, and, in the case of blood pressure, obtaining baseline readings in case of a medical emergency. It is important to emphasize, however, that we do not, within our scope of practice, specifically measure blood pressure as part of overall secondary preventive medical care. Likewise, the importance we place on blood glucose control relates directly to the impact of poorly controlled diabetes on a patient's ability to tolerate dental treatment (e.g., cardiovascular status, hypoglycemia) and the effects of elevated blood glucose on wound healing and response to periodontal therapy, to name just a few. Again, the fact that dental providers are adept, in many cases, at identifying patients with diabetes or prediabetes based on their dental presentation and non-dental clinical signs and symptoms does not argue in favor of providing routine primary care point of care testing for all asymptomatic patients.

Accepting the argument that the rationale for performing certain screening procedures relates directly to our ability to provide appropriate patient care does not negate the obligation, when we identify concerns not directly within our scope of practice, that we must advise our patients that follow-up with their primary medical provider is appropriate. For example, while a patient's blood pressure may be within an acceptable range to provide dental treatment based on the dental provider's assessment of all risk factors, when higher than ideal for long-term cardiovascular health, a referral to the patient's primary care medical provider is mandated.

With respect to primary preventive care, dentists have a professional responsibility to advocate for lifestyle changes that positively impact overall health, especially as these may relate to our broad area of expertise. A dentist who asks his or her patient about the use of seatbelts and safe firearm storage is clearly within the scope of practice, as failure to use seatbelts and improper storage of firearms are both associated with an increased risk of maxillofacial trauma and death. ${ }^{37}$ Likewise, the dentist who discusses the benefits of MMR, high risk HPV, varicella zoster, or shingles vaccinations with a patient and, where appropriate, recommends that the patient schedule an appointment with his or her primary care medical provider to receive these vaccinations, should be commended. This should be a part of every conscientious dentist's health counselling. There is nothing innovative about this approach; this is the current standard of care in dentistry.

However, the fact that many preventable infections can directly impact the oral and maxillofacial complex does not, in and of itself, argue in favor of dental providers' taking on the role of the primary care medical provider, such as administering routine immunizations in the dental setting. We are not arguing that dentists should not be called upon, in the case of a public health emergency, to offer our expertise. In fact, one of the authors (PCE) participated in a pilot study several years ago that demonstrated dental faculty members and students were ideally suited to help administer vaccinations in a university-run mass vaccination clinic. Instead, it is our contention that dental providers should not be selectively offering LPPC procedures, such as routine immunizations or ordering screening tests outside our scope of practice in the absence of a comprehensive primary medical evaluation and long-term follow-up.

The suggestion that some patients see their dental provider more frequently than their primary care medical provider is not, in and of itself, a valid argument for providing these primary care services. Likewise, the rationale for providing these services must not be to replicate the role of the patient's primary medical provider in order to manage any perceived or predicted shortages of primary medical care providers. There clearly are more cost-effective alternatives to addressing any such shortages, such as expanding the class sizes of medical and non-MD/ DO schools (e.g., nurse practitioners, physician assistants, pharmacists). 
In response to our concerns about the risks associated with a fragmentary approach to primary medical care, in which dental providers pick and choose which aspect of primary care medicine to offer their patients, we have heard the argument made that appropriately trained dental providers could in fact provide the full spectrum of primary medical care. This position, by necessity, would advocate for the training of dental providers who are not only capable of offering all aspects of dental treatment, including routine preventive dentistry, prosthodontics, orthodontics, endodontics, periodontics, pediatric dentistry, radiology, and clinical oral pathology, but also comprehensive primary medical care. This argument fails to recognize the incredible growth in biomedical knowledge over the past several decades and how, as a direct result of this increasing knowledge base, scientific progress in medicine and dentistry has been characterized by increasing specialization during this period. While 100 or more years ago, family physicians, with occasional assistance from their general surgery colleagues, managed patients from cradle to grave, it is increasingly difficult to keep up with the breadth of knowledge needed in medicine and dentistry. Even within individual specialties, the move towards greater subspecialty training is clear. This trend is even evident among nurse practitioners and physician assistants, who are choosing specialty practice over primary care..$^{38}$ There is clear evidence that referral to appropriate specialists for diagnosing and managing less common, more challenging, or more significant medical conditions portends both better patient care ${ }^{39}$ and cost savings for health care payers. In light of this trend towards increased specialization, we do not see how expanding the dentist's scope of practice to include comprehensive non-oral and maxillofacial primary care medicine ultimately benefits our patients.

We also believe that advocating for dental care providers to expand into primary medical care does not represent a valid approach to dealing with issues of dental workforce "busyness." Resolving these dental human resource issues will require a much larger dialogue involving CODA, dental practitioners, allied dental professionals, and dental schools. Regardless, it is more probable than not that any additional revenue generated through provision of non-dental primary care medicine will be dwarfed by the additional cost of support staff and frustrations associated with medical billing, ${ }^{40}$ not to mention questions of increasing regulatory burden and potential liability.

\section{Improvements to the Status Quo}

These comments notwithstanding, we are by no means advocating for the status quo. It remains our contention that it is critically important that tomorrow's dental students graduate with a much greater breadth and depth of knowledge with respect to the fundamentals of clinical medicine: an education that complements and builds on systems-based biomedical science instruction. But the reality is that, at many dental schools, students receive extensive didactic education in the biomedical sciences, usually in isolation from clinical dental medicine, during the first year or two of the predoctoral curriculum, with much of this knowledge not translating into direct patient care and clinical practice. Not only is this a tremendous lost opportunity; this fragmented approach to dental education does not support the progress dentistry has seen from a technically based apprenticeship to a respected health profession. There are many dynamics at play: a heavy emphasis on accumulating technical experience in order to graduate; increased administrative burden resulting from increasing state and government regulations; and, in some cases, dental school faculty members for whom clinical medicine is outside their realm of comfort and who lack access to effective faculty development programs in this area.

We believe that a critical element in integrating preclinical biomedical education into the later clinical education is the availability of high-quality experiential clinical clerkships in primary care medicine. The objective of this broad-based medical education is not to train dental providers to serve as primary care medical providers, but to ensure that tomorrow's dental graduates possess the prerequisite knowledge and vocabulary to be able to function effectively, and as an equal partner, in the health care team of tomorrow. ${ }^{41}$ If we are to advocate for a greater emphasis on interprofessional education in the health sciences, it is critical that dental students be able to both "talk the talk and walk the walk." There could be nothing more damaging to the prospect of building greater partnerships among the health disciplines than pushing head first into interprofessional education without first ensuring that our dental students have a solid background in clinical medicine.

But again, the intent is not to train dentists of the future to provide routine non-dental primary medical care. Dentists should not be providing routine vaccinations, ${ }^{42}$ ordering metabolic profiles or bone density scans, or referring patients for mam- 
mography and colonoscopies, unless the rationale for ordering or providing these procedures is related to our scope of practice. As we have previously argued, there already are a large number of conditions clearly within the scope of our education, ranging from management of chronic vesiculobullous lesions to treatment of preneoplastic epithelial changes that primary dental care providers are best suited to manage; yet in many cases, patients with these conditions are currently being referred out to non-dental providers. ${ }^{43}$ In light of this, why would we contemplate expanding outside our area of expertise until first taking full ownership of these neglected areas that are already within our expertise? ${ }^{41}$

Another critical question that must be addressed is the financial impact of any proposed changes in how health care is delivered. Duplication of services, ${ }^{44}$ such as ordering routine metabolic profiles on the otherwise asymptomatic patient in the dental setting, will only serve to drive up health care costs, particularly in the absence of a centralized electronic medical record (EMR) shared among all health care providers. Despite provisions in the Affordable Care Act (ACA) to encourage adoption of EMRs, the lack of interoperability among the many proprietary EMR formats does not facilitate the sharing of information between health care providers. Furthermore, we are very concerned that encouraging our dental graduates to conduct non-dental primary preventive medical care for their patients simply because these patients may traditionally visit their dentist more frequently than their physician could further exacerbate the fragmentation of care that is already a significant issue in our health care system.

But the most important consideration is how changes to health care delivery impact patient outcomes. We have all seen many examples in which patients have drifted through the health care system, often accompanied by unnecessary and costly tests, in search of an otherwise simple diagnosis. ${ }^{45}$ It is our contention that time spent focusing on management of conditions clearly within the scope of the dental provider, such as erosive lichen planus, is a more valuable use of the limited educational time in the dental curriculum than trying to educate dental students in the complexities of non-oral health-related primary care medicine.

Overall, while we absolutely agree that graduating dentists must have a solid theoretical and practical knowledge of primary care medicine in order to safely and effectively provide oral health care, including preventive medical care, within the scope of their training, we see no logical rationale for suggesting that dentists should routinely provide LPPC. Instead, we should direct our efforts at graduating dentists who are fully capable of and comfortable with diagnosing and managing the large number of relatively common non-tooth-related pathologic conditions of the oral and maxillofacial complex, such as erosive lichen planus and preneoplastic epithelial conditions, that in many cases are not currently being managed in the dental setting.

\section{Response by Drs. Giddon and Donoff to View- point 2:}

Our arguments support change in the scope of practice for the dentist to improve the health of patients. The designation "oral physician" simply describes a dentist who not only learned about the importance of taking care of the whole patient, but also practices this philosophy. It is not about an occasional finger stick, but an attitude and practice philosophy that comes from didactic learning and hands-on experiences in dental school. It is not about an occasional flu vaccination, but changing the scope of dental practice. We do not advocate graduating a dentist of intermediate competence. In fact, the current dental graduate has excellent competence as judged by meeting requirements of graduation and passing live patient examinations. These same competent graduates have minimal experiences using the knowledge gained about the basis of human disease and its management in school. The need for more general practice residencies speaks to this problem.

Viewpoint 2's statements that current dental education fosters medical inquiry is misleading. Even if the medical basis of dental practice is taught in the first two years of most dental schools, it is forgotten in the deluge of dental procedures because it is neither integrated nor reinforced throughout the preparation for dental practice. Finally, we are practical and acknowledge that nothing in the workforce will change until the interprofessional and other barriers to reimbursement are eliminated. Also, a dual degree with licensure in both dentistry and medicine is an option, particularly for those who take a leadership role in the proposed changes.

\section{Response by Drs. Edwards and Goldblatt to Viewpoint 1:}

We agree with Viewpoint 1's premise that today's dental school graduate must be fully knowledgeable in all aspects of primary medical care that impact our ability to care for our patients, but 
we differ with respect to the rationale and desired outcome of this knowledge. We completely agree with the importance of expanding cooperation with our counterparts in related health care specialties, recognizing the overarching goal of improving patients' overall health and well-being. However, we respectfully disagree that there should be an additional level of primary dental provider, in addition to the current dental specialties: specifically, what they refer to as a new class of oral physicians as differentiated from what they designate the traditional dentist. Instead, we contend that today's graduating dentist already has the knowledge base to effectively provide all those aspects of primary care medicine that fall within our scope of practice. We also believe that today's dental students should graduate with the experiential training required to function at the level designated "oral physician" by Viewpoint 1. In our opinion, this represents the minimum requirement to function as a competent dentist.

We are concerned that offering piecemeal primary medical care diagnostic testing and preventive procedures outside our scope of practice will only serve to further fragment the health care system. Instead, we should concentrate on providing dental students with strong clinical experience in the numerous areas that currently fall within our scope of practice and for which dentists already have the greatest theoretical knowledge, but that are, in many cases, not being managed by dental providers. Examples include the long-term management of patients with erosive lichen planus and patients presenting with potentially preneoplastic epithelial lesions and sleep medicine appliance therapy, to name just a few. Our contention remains that we should first ensure, within current dental curricula, expanded access to high-quality clinical experiences that focus on those overlooked areas already within our scope of practice. Until this critical milestone is reached, we see no compelling argument justifying the need for a new class of dental providers who will expand into areas outside our scope of practice.

\section{Disclosures}

The opinions expressed in this Point/Counterpoint are those of the authors and in no way reflect the policy of the authors' employers.

\section{REFERENCES}

1. Tanaka K, Honda T, Kitamura K. Dentistry in Japan should become a specialty of medicine with dentists educated as oral physicians. J Dent Educ 2008;72(9):1077-83.
2. Jones NJ, ed. Transforming Harvard School of Dental Medicine: 2014 dean's report. In: Harvard School of Dental Medicine. Boston: Puritan Press, 2014.

3. Feld MJ, ed. Dental education at the crossroads: challenges and change. An Institute of Medicine Report. Washington, DC: National Academies Press, 1995.

4. Gies WJ. Dental education in the United States and Canada: a report to the Carnegie Foundation for the Advancement of Teaching. New York: Carnegie Foundation for the Advancement of Teaching, 1926.

5. Nasseh K, Vujicic M, Glick M. The relationship between periodontal interventions and health care costs and utilization: evidence from an integrated dental, medical, and pharmacy commercial claims database. Health Econ 2017;26(4):519-27.

6. Nasseh K, Greenberg B, Vujicic M, Glick M. The effect of chairside chronic disease screenings by oral health professionals on health care costs. Am J Public Health 2014;104(4):744-50.

7. United Health Care. Medical dental integration study. At: www.uhc.com/content/dam/uhcdotcom/en/Private $\% 20$ Label\%20Administrators/100-12683\%20Bridge2Health Study_Dental_Final.pdf. Accessed 16 Feb. 2016.

8. Hedayatnia S, Giddon DB. A business case for oral physicians: market analysis and potential practice models for dentists to address the United States primary care shortage. At: www.hmsreview.org/?article =a-business-case-for-oral-physicians-market-analysisand-potential-practice-models-for-dentists-to-addressthe-united-states-primary-care-shortage. Accessed 27 Sept. 2015.

9. Giddon DB, Swann B, Donoff RB, Hertzman-Miller R. Dentists as oral physicians: the overlooked primary health care resource. J Prim Prev 2013;34(4):279-91.

10. Association of American Medical Colleges. Physician shortages to worsen without increases in residency training. At: www.aamc.org/download/150584/data/physician_shortages_factsheet.pdf. Accessed 1 Dec. 2015.

11. Steinbrook R. Easing the shortage in adult primary care: is it all about money? N Engl J Med 2009;360(26):2696-9.

12. Strauss SM, Alfano MC, Shelley D, Fulmer T. Identifying unaddressed systemic health conditions at dental visits: patients who visited dental practices but not general health care providers in 2008. Am J Public Health 2012;102(2):253-5.

13. Greenberg BL, Kantor ML, Jiang SS, Glick M. Patients' attitudes toward screening for medical conditions in a dental setting. J Public Health Dent 2012;72(1):28-35.

14. Thomas R. Herodotus in context: ethnography, science, and the art of persuasion. New York: Cambridge University Press, 2002.

15. Gordon S, Berg R, Park S, et al. Shaping tomorrow's oral physicians: two approaches to curricular reform. Symposium at American Dental Education Association Annual Session, March 14, 2016, Denver, CO.

16. Giddon DB. Mental-dental interface: window to the psyche and soma. Perspect Biol Med 1999;43(1):84-97.

17. Giddon DB. Need for three levels of oral health providers: oral physician, dentist, and dental therapist. Presentation at American Dental Education Association Annual Session, March 2017, Long Beach, CA. 
18. Nash D. The oral physician: creating a new oral health professional for a new century. J Dent Educ 1995;59(5): 587-97.

19. Giddon DB. Should dentists become "oral physicians"? J Am Dent Assoc 2004;135:438-49.

20. Guay AH. Where is dentistry going? (editorial). J Am Dent Assoc 2016;147(11):853-5.

21. Bader JD. Challenges in quality assessment of dental care. J Am Dent Assoc 2009;140(12):1456-64.

22. Dang C. Willingness of Rhode Island dentists to provide limited primary care. Providence, RI: Department of Public Health, Brown University, 2015.

23. Catlett A. Attitudes of dental hygienists towards independent practice and professional autonomy. J Dent Hyg 2016;90(4):249-56.

24. Hanson SH. Speaking out against more certifications for PAs. At: www.physicianspractice.com/blog/speaking-outagainst-more-certifications-pas?cid=TP2. Accessed 6 Oct. 2016.

25. Nicholson JG. Physician assistant malpractice history: comparing PAs to physicians and nurse practitioners At: expertpages.com/news/Physician_Assistant_Malpractice History.htm. Accessed 7 Oct. 2016.

26. Personal communication with Dr. Leon Assael, 18 Dec. 2015.

27. Bertolami CN. Access to dental care: is there a problem? Am J Public Health 2011;101(10):1817.

28. Compilation of the Social Security Laws. Part E: Miscellaneous Provisions. Definitions of services, institutions, etc. [442]. Sec. 1861. [42 U.S.C. 1395x].1861(r).

29. Rosebury T. On bulletin number nineteen: thirty years later. In: Orlando FJ, ed. William John Gies: his contribution to the advancement of dentistry. New York: William J. Gies Foundation for the Advancement of Dentistry, 1992.

30. Minnesota Department of Health, Office of Rural Health and Primary Care. Early impacts of dental therapists in Minnesota: report to the Minnesota legislature. St. Paul: Minnesota Department of Health, 2014.

31. Palmer C. ADA reviews draft Senate legislation. At: www. ada.org/en/publications/ada-news/2012-archive/june/adareviews-draft-senate-legislation. 2012. Accessed 14 June 2016.

32. VCU SOD receives $\$ 2.4$ million grant to improve dental care access and interprofessional training. At: www.adea. org/BDEBlog. aspx?id=27932\&blogid=27619\#sthash. rQVO1Vuk.dpuf. 2015. Accessed 14 Dec. 2015.

33. Edwards PC. Considering that this is such a rare condition, should we really be expected to recognize it? Oral Surg Oral Med Oral Pathol Oral Radiol Endod 2011;112(2):143-5.
34. Olson CM, Burda BU, Beil T, Whitlock EP. Screening for oral cancer: a targeted evidence update for the US Preventive Services Task Force: evidence synthesis no. 102. AHRQ Pub. No 13-05186-EF-1. Rockville, MD: Agency for Healthcare Research and Quality, 2013.

35. Edwards PC. Oral cancer screening for asymptomatic adults: do the United States Preventive Services Task Force draft guidelines miss the proverbial forest for the trees? Oral Surg Oral Med Oral Pathol Oral Radiol 2013;116(2):131-4.

36. Edwards PC. Expanding the scope of practice of dental medicine for tomorrow's dental graduate: a unique role for our combined specialties. Oral Surg Oral Med Oral Pathol Oral Radiol 2014;118(2):143-5.

37. Some states have, inexplicably, passed legislation aimed at restricting a health care provider's ability to discuss firearm safety with patients. AMA J Ethics 2014;16:284-8.

38. Hooker RS, Everett CM. The contributions of physician assistants in primary care systems. Health Soc Care Community 2012;20(1):20-31.

39. Badheka AO, Patel NJ, Grover P, et al. Impact of annual operator and institutional volume on percutaneous coronary intervention outcomes: a 5-year United States experience (2005-09). Circulation 2014;130(16):1392406.

40. Casalino LP, Nicholson S, Gans DN, et al. What does it cost physician practices to interact with payers? In: Young PL, Olsen LA, eds. The health care imperative: lowering costs and improving outcomes. Workshop series summary. Washington, DC: National Academy of Sciences, 2010:151-9.

41. Nielsen M, Gibson L, Buelt L, et al. The patient-centered medical home's impact on cost and quality: review of evidence, 2013-14. At: www.pcpcc.org/resource/patientcentered-medical-homes-impact-cost-and-quality. Accessed 1 Apr. 2016.

42. Glick M. Why don't dentists provide immunizations? A missed opportunity. J Am Dent Assoc 2013;144(10): 1098-100.

43. Edwards PC. The future of dental education: toward disruptive innovation or incremental improvements? Oral Surg Oral Med Oral Pathol Oral Radiol 2015;119(3): 257-9.

44. Owens MK. Costs of uncoordinated care. In: Young PL, Olsen LA, eds. The health care imperative: lowering costs and improving outcomes. Workshop series summary. Washington, DC: National Academy of Sciences, 2010:131-40.

45. Edwards PC. Persistent mouth sores: working with your oral health care provider to reach a diagnosis. 2016. At: www. pemphigus.org/persistent-mouth-sores-working-withyour-oral-health-care-provider-to-reach-a-diagnosis/. Accessed 1 Apr. 2016. 